Ocular Oncology

and Pathology
Ocul Oncol Pathol 2018;4:261-266

DOI: 10.1159/000485978
Received: August 15, 2017

Accepted after revision: December 1, 2017

Published online: February 1, 2018

\title{
Eye-Sparing Treatment for Diffuse Invasive Conjunctival Melanoma
}

\author{
Leandro J. Chaves $^{a}$ Bradley Huth ${ }^{b}$ James J. Augsburger ${ }^{c}$ Zelia M. Correa ${ }^{c}$ \\ a Department of Ophthalmology, Otorhinolaryngology, and Head and Neck Surgery, Ribeirão Preto Medical School, \\ University of São Paulo, Ribeirão Preto, Brazil; ${ }^{b}$ Department of Radiation Oncology, University of Cincinnati College \\ of Medicine, Cincinnati, $\mathrm{OH}, \mathrm{USA}$; $^{\mathrm{C}}$ Department of Ophthalmology, University of Cincinnati College of Medicine, \\ Cincinnati, OH, USA
}

\section{Established Facts}

- Orbital exenteration has not demonstrated significant improvement in survival rates of patients with invasive conjunctival melanoma.

- Different combined therapies for diffuse/invasive and partially excised conjunctival melanoma may achieve tumor control if properly indicated.

\section{Novel Insights}

- Combining multiple conservative treatments enables local tumor control in some patients with diffuse invasive conjunctival melanoma.

- Meeting patients' needs and beliefs may improve communication and compliance when managing advanced tumors.

\section{Keywords}

Melanoma, eye · Melanoma, conjunctiva · Brachytherapy · Enucleation

\section{Abstract}

The management of patients with diffuse invasive conjunctival melanoma focuses on local tumor control and screening for metastasis. Despite the lack of consensus on the benefit of sentinel lymph node biopsy for these neoplasms, the information obtained by histopathology is useful for tumor staging and treatment planning. Due to the lack of evidence of survival improvement, orbital exenteration is being performed with diminishing frequency. We describe a patient with diffuse invasive conjunctival melanoma and lymph node involvement treated by tumor debulking, brachytherapy (custom unshielded radioactive device), and adjuvant ipilimumab who has had a favorable outcome without emergence of local tumor relapse or distant metastasis during 16 months of follow up.

(c) 2018 S. Karger AG, Basel

\section{Introduction}

Historically, orbital exenteration has been recommended for extensive conjunctival malignant melanoma invading the orbit, palpebral margin, fornices, and/or

\section{KARGER}

(c) 2018 S. Karger AG, Basel

E-Mail karger@karger.com

www.karger.com/oop
Zelia M. Correa, $\mathrm{MD}, \mathrm{PhD}$

Department of Ophthalmology, Medical Science Building 5th floor 231 Albert Sabin Way

Cincinnati, OH 45267-0527 (USA)

E-Mail correazm@uc.edu 

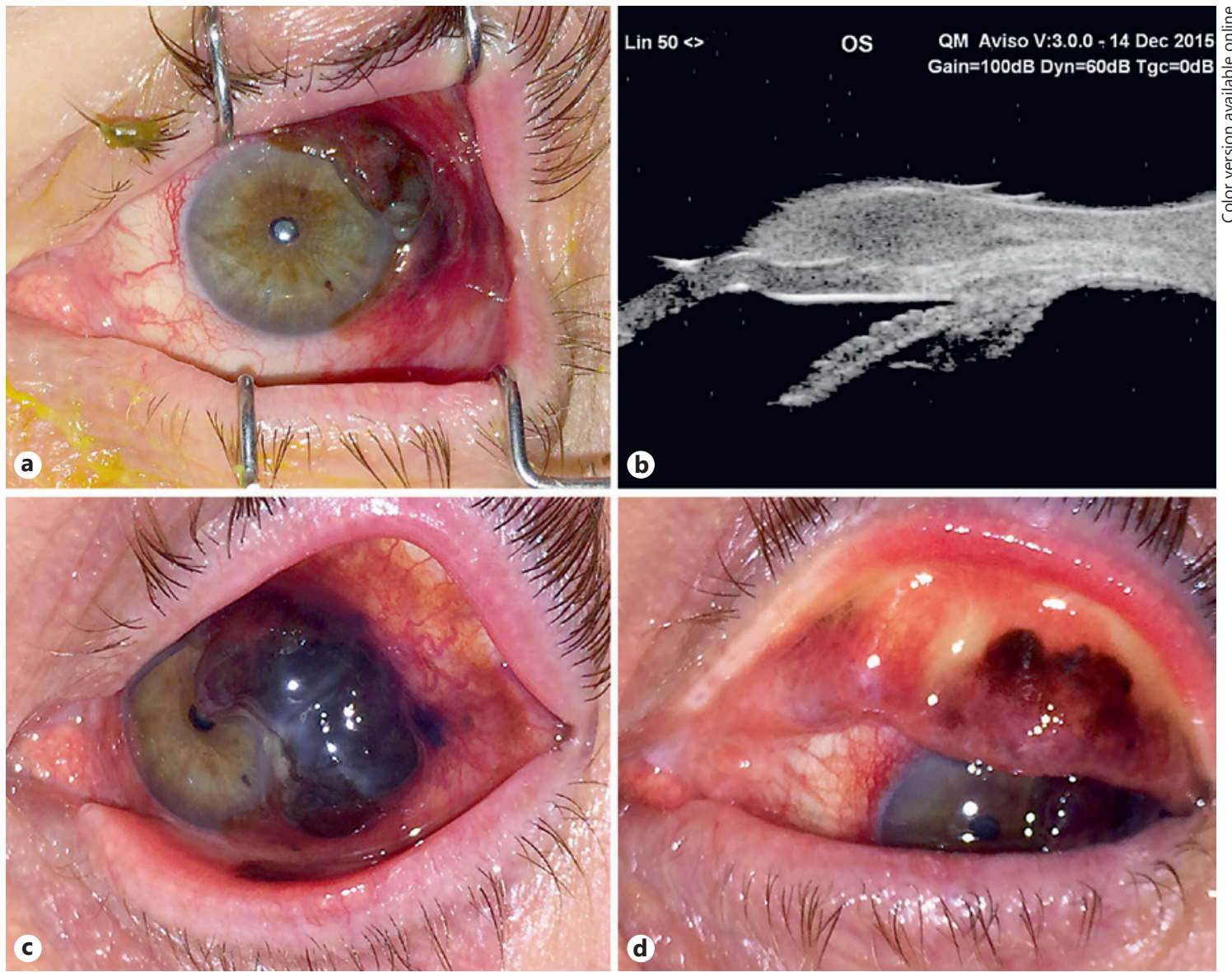

Fig. 1. Baseline and pretreatment clinical findings left eye (OS). a Baseline clinical presentation showing multifocal nodular conjunctival melanoma OS. b Ultrasound biomicroscopy OS shows a prominent nodular epibulbar mass straddling the limbus superotemporally and bowing the cornea. The mass exhibits homogeneous low to moderate sonoreflectivity throughout. c Clinical presentation pretreatment (3 months after initial visit) showing marked tumor progression on the bulbar conjunctiva. d Clinical presentation pretreatment showing tumor invading the tarsal conjunctiva.

caruncle. Unfortunately, this disfiguring procedure has failed to demonstrate significant improvement in patient survival compared to other conservative treatments [1]. The goals of treatment of diffuse conjunctival melanoma $(\mathrm{CM})$ are local tumor eradication and metastatic assessment. We describe a patient with extensive CM and preauricular lymph node involvement managed by eye-sparing therapies.

\section{Case Report}

A 72-year-old man complained of irritation, foreign body sensation, profuse clear discharge, and crusting of the left eye (OS) for 8 weeks. An outside eye care provider had noted a "darkbrown pigmented" lesion OS thought not to be concerning 6 years earlier. At our examination, visual acuity was $20 / 25^{+}$in the right eye (OD) and 20/40- OS. Examination OD showed completely normal findings. In contrast, examination OS revealed an extensive dark-brown conjunctival tumor involving the bulbar, forniceal, and palpebral conjunctiva (Fig. 1a). The tumor had an accentuated nodular portion overlying the superior and temporal limbus measuring $20.0 \times 12.0 \mathrm{~mm}$ in basal diameter and $2.5 \mathrm{~mm}$ in thickness and ill-defined diffuse involvement of the superior and inferior fornices and tarsal conjunctiva. There were no palpable preauricular or cervical lymphadenopathies. Ultrasound biomicroscopy OS revealed a solid homogeneous epibulbar tumor with low internal reflectivity causing focal bowing of the limbus and cornea (Fig. 1b). Our clinical diagnosis was diffuse multifocal primary CM involving bulbar and palpebral conjunctiva OS arising from primary acquired melanosis of the conjunctiva.

Management options were discussed with the patient and included primary lid-sacrificing left orbital exenteration, partial excision (debulking) of the tumor followed by either intensive topical chemotherapy (e.g., mitomycin C or 5-fluorouracil) or immuno- 

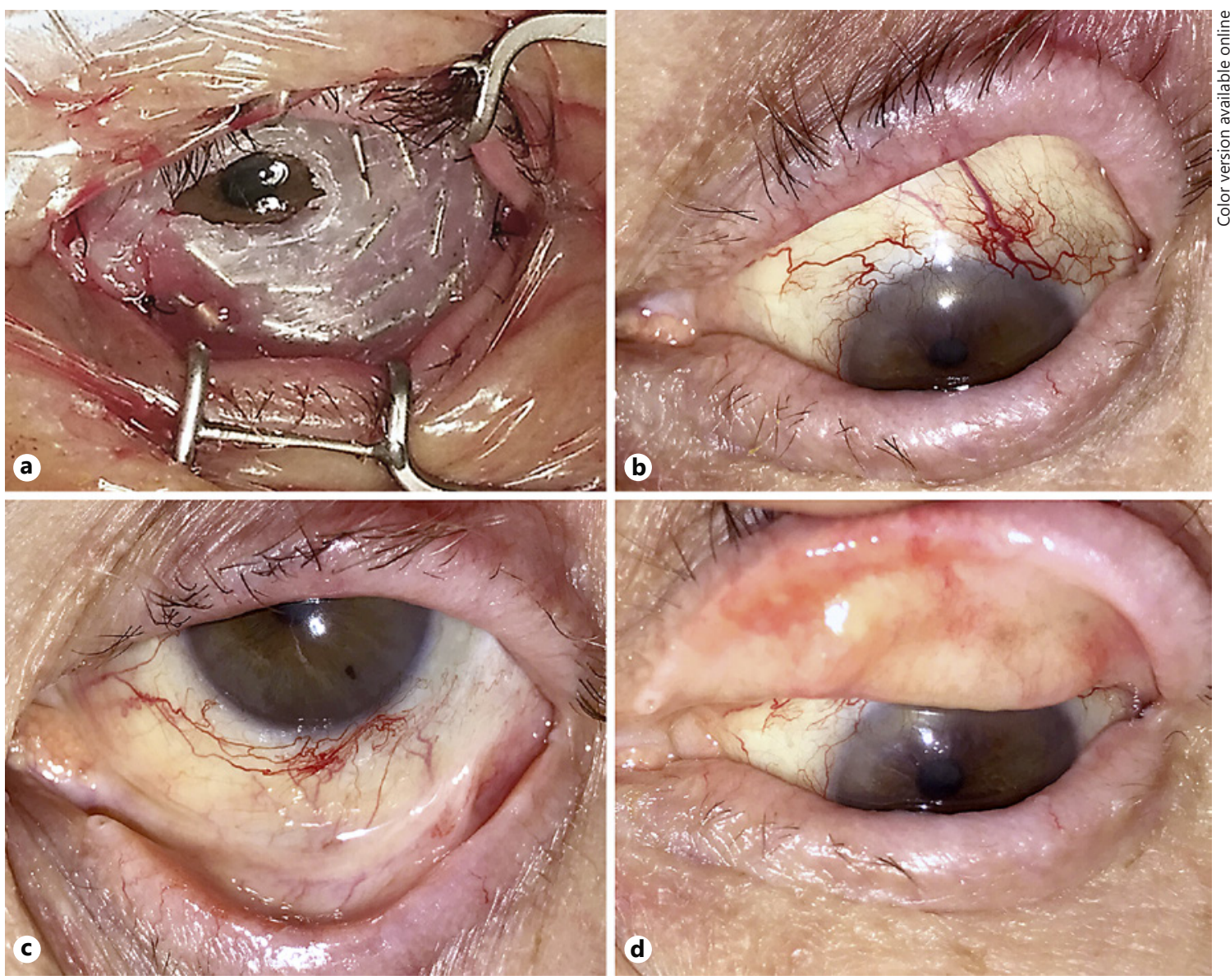

Fig. 2. Iodine-125 unshielded plaque radiation and patient clinical outcome. a Custom unshielded radioactive iodine-125 device covering the ocular surface and fornices. b-d Clinical outcome 13 months after radiation treatment. There were no signs of local recurrence but the patient presented with peripheral corneal vascularization on the superior limbus and a partial lash loss.

therapy (e.g., interferon alpha 2B), and possibly external beam radiation or unshielded plaque radiotherapy as well.

The patient refused to commit to either method of treatment initially. He returned 3 months later reporting deterioration of vision OS (20/50) and tumor progression (Fig. 1c, d). The tumor measured $22.5 \times 11.5 \mathrm{~mm}$ in basal diameter and $5.0 \mathrm{~mm}$ in thickness. The patient adamantly refused exenteration but consented to debulking of his tumor with sentinel lymph node (SLN) biopsy followed by treatment of his residual conjunctival melanocytic tissue with a custom unshielded radioactive iodine-125 device. The debulking entailed removal of the nodular portion of the tumor and advancement with simple closure of the conjunctiva.

The patient was brought to the operating room for tumor debulking and SLN biopsy under general anesthesia. After successful debulking of the nodular surface tumor, a the template for the unshielded device was custom-fitted in the patient's ocular surface from a silica gel sheet $(2.0 \mathrm{~mm}$ thick) stretching the superior and inferior fornices in order to deliver the planned radiation. Biopsy of SLN was performed using lymphoscintigraphy and radio-guid- ed SLN mapping and biopsy. There were no complications. The patient tolerated both the anesthesia and surgery, and recovered promptly.

Two weeks later, the patient was brought back to the operating room for the planned second step of the treatment under retrobulbar block plus monitored anesthesia care. The fitted silica molded device received iodine-125 seeds that were distributed to deliver a planned dose of 32.0 Gy to the tumor apex and 33.5 Gy to the sclera in $23 \mathrm{~h}$. Over the iodine-125 seeds, another thin layer of silica gel was glued in order to seal the radioactive seeds. Tumor thickness (i.e., apical height was calculated using measurements from ultrasound biomicroscopy and CT of the orbits. The 24 radioactive seeds provided $1.325 \mathrm{~Gy} / \mathrm{h}$ to the tumor apex and $1.395 \mathrm{~Gy} / \mathrm{h}$ to the sclera during the treatment. Surgical implantation was followed by a temporary tarsorrhaphy to ensure stability of the radioactive implant (Fig. 2a). The patient was admitted for 23-hour observation followed by surgical removal and hospital discharge.

Histopathology confirmed invasive epithelioid CM, extending to the inked peripheral and deep margin (Fig. 3a, b). SLN biopsy 

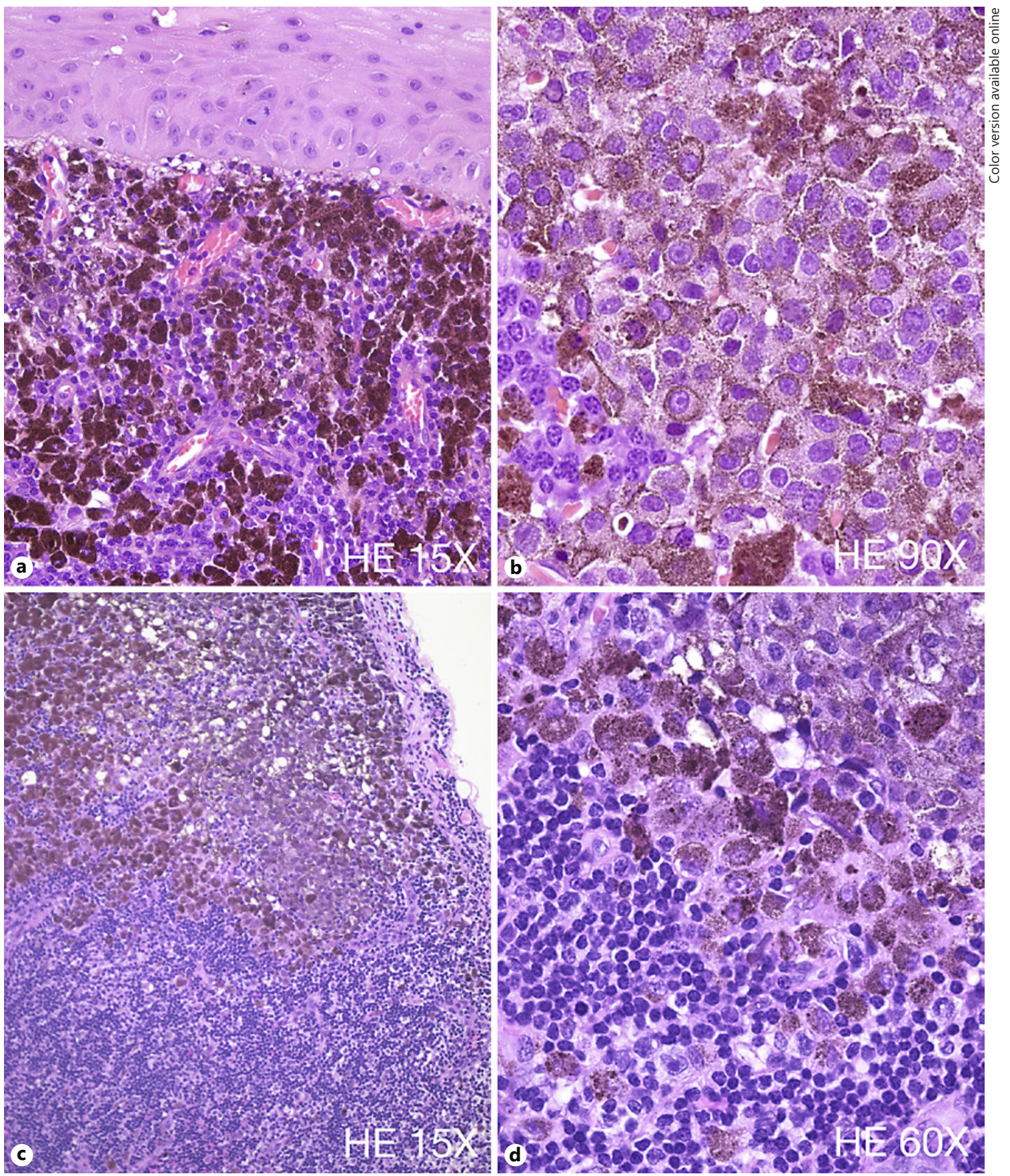

Fig. 3. Histopathology findings. a Microscopic examination of the excised conjunctiva reveals a subepithelial proliferation of hexagonal-shape melanocytes with high nuclear/cytoplasmic ratio and very large nuclei. $\mathbf{b}$ These cells present a very irregular chromatin and frequent mitotic figures are noted. c, d Sentinel lymph node biopsy shows pigmented melanocytic cells with very atypical features consistent with epithelioid melanocytes and similar to the cells found in the conjunctival specimen. 
revealed melanoma within one of the sampled preauricular nodes (Fig. 3c, d). Due to the histopathology findings, additional left neck dissection was recommended. The dissection was performed 2 weeks after the radiation and involved a preauricular incision and elevated flaps over the mandible and over levels I, II, and III of the neck. All 14 lymph nodes and superficial parotid tissue removed were negative on histopathology. The patient had a drain placed in his neck for 3 days after surgery. There were no complications.

Systemic workup included complete physical examination and positron-emission tomography (PET) imaging that failed to reveal any findings suggestive of metastasis. His cancer staging based on the American Joint Committee on Cancer 7th edition staging was T3bN1M0. Considering the multifocal pattern of the conjunctival tumor and positive SLN, the oncologist recommended 4 cycles of adjuvant therapy with ipilimumab $3 \mathrm{mg} / \mathrm{kg}$ every 3 weeks for 4 doses. Treatment was well tolerated but he reported mild fatigue that did not warrant interruption of planned therapy and ceased after the last treatment cycle.

The patient is currently 16 moths post-radiation treatment showing satisfactory response to treatment and excellent local tumor control. His visual acuity is 20/25 OD and 20/80 OS. He has partial lash loss, dry eye, peripheral corneal vascularization superiorly, secondary glaucoma (controlled with topical medications) and radiation-induced posterior subcapsular cataract. Fundus exam OS remains normal. He has no evidence of regional nodal recurrence or distant metastasis on biannual PET imaging (Fig. 2bd).

\section{Discussion}

Because exenteration has shown no survival benefit for patients with extensive/invasive CM over that of less aggressive treatment methods, alternative globe-sparing treatments are currently being offered to some patients diagnosed with this neoplasm [2-4].

Our patient's tumor had clinical features suggestive of high metastatic risk (multifocal presentation and thickness) prompting our recommendation to perform SLN biopsy despite the lack of consensus on indication and impact on survival [1-6]. Savar et al. [2] suggested that increased tumor thickness and microscopic ulceration in CM may be important predictors of tumor aggressiveness and regional lymph node metastasis. Despite the increased tumor thickness, there was no evidence of tumor ulceration [2]. Nevertheless, SLN biopsy identified metastasis to one preauricular lymph node. We used an unshielded iodine-125 device to treat the residual tumor because we needed to treat the fornices and the tarsal conjunctiva simultaneously. By using a superficial radioactive source, we hoped to limit the radiation exposure of the fundus, lacrimal gland, and orbit. Despite the patient having developed moderate dry eye and a radiation-induced cataract, he still has good vision potential in that eye.

Eye-Sparing Treatment for Conjunctival

Melanoma
Different local adjuvant therapies can be used in cases of partially excised tumors, including conjunctival cryotherapy, topical mitomycin C [2-4], 5- fluorouracil, or interferon alpha- $2 \mathrm{~B}$ eye drops, and radiotherapy (external beam, proton beam, and brachytherapy) $[2,5]$. Because we knew complete excision was not achievable for this patient and he was unlikely to be compliant with topical therapy, unshielded iodine-125 radiotherapy was thought to be a good choice given its potential to provide local tumor control with limited secondary side effects.

Following the radiotherapy, additional neck dissection, and PET imaging, the oncologist discussed with the patient the options for adjuvant therapy in the setting of high risk for recurrence of his CM. If this patient had cutaneous melanoma with similar features he would qualify for adjuvant interferon. However, the evidence is not strong for CM and the side effects would not be worth it. After discussing with other centers, the recommendation was for adjuvant systemic treatment with ipilimumab. This off-label treatment is based on phase III trial data from cutaneous melanoma, which showed an increase in relapse-free survival with tolerable treatment-related adverse events [7]. Our patient agreed to the off-label treatment but not to being randomized to a trial. He also chose not to have additional genetic testing. Despite of that, we recognize the lack of effectiveness data on using ipilimumab specifically for CM.

Regarding posttreatment follow-up, he currently has moderate to severe dry eye, lash loss, shortening of the inferior fornix, and radiation-induced cataract. All of these are manageable at this time. Fortunately, he received a very low dose to his retina ( $5 \mathrm{~Gy}$ ) and fovea/disc $(>1 \mathrm{~Gy})$ making it unlikely for him to develop radiation retinopathy.

In summary, our patient with diffuse advanced CM with SLN involvement is tumor free 16 months after partial excision, brachytherapy, and adjuvant ipilimumab. We plan to continue periodic multidisciplinary follow-up biannually to monitor local recurrence or metastasis, and potential side effects of radiotherapy.

\section{Acknowledgements}

This work was supported in part by the James J. Augsburger Ocular Oncology of the Department of Ophthalmology, College of Medicine, University of Cincinnati; the Dr. E. Vernon and Eloise C. Smith Chair of Ophthalmology Fund, Department of Ophthalmology, College of Medicine, University of Cincinnati (chairholder: James J. Augsburger); and Dr. Mary Knight Asbury Chair of Ophthalmic Pathology and Ocular Oncology Fund, Department of Ophthalmology, College of Medicine, University of Cincinnati (chairholder: Zelia M. Correa). 


\section{Statement of Ethics}

The patient described herein has given his informed consent for this publication and our institute's committee on human research has provided a waiver for retrospective data reporting for the Ocular Oncology Service.

\section{Disclosure Statement}

Leandro J. Chaves, Bradley Huth, and James J. Augsburger have no financial conflicts of interest to disclose. Zelia M. Correa is a consultant for Castle Biosciences, Inc.

\section{References}

1 Shields CL, Chien JL, Surakiatchanukul T, Sioufi K, Lally SE, Shields JA: Conjunctival tumors: review of clinical features, risk, biomarkers, and outcomes - the 2017 J. Donald M. Gass lecture. Asia Pac J Ophthalmol 2017; 6:109-120.

2 Savar A, Esmaeli B, Ho H, Liu S, Prieto VG: Conjunctival melanoma: local-regional control rates, and impact of high-risk histopathologic features. J Cutan Pathol 2011;38:18-24.

3 Dzwonczyk AM, Corrêa ZM, Augsburger JJ, Kulwin D: Eye-preserving treatment for invasive conjunctival melanoma. JAMA Ophthalmol 2014;132:1486-1488.
4 Kim EC, Kim MS, Kang NY: Excision with corneoscleral lamellar keratoplasty and amniotic membrane transplantation of a corneal displaced recurrent conjunctival melanoma. Korean J Ophthalmol 2012;26:383-387.

5 Mendoza PR, Grossniklaus HE: Sentinel lymph node biopsy for eyelid and conjunctival tumors: what is the evidence? Int Ophthalmol Clin 2015;55:123-136.
6 Vora GK, Demirci H, Marr B, Mruthyunjaya $\mathrm{P}$ : Advances in the management of conjunctival melanoma. Surv Ophthalmol 2017;62:2642.

7 Eggermont AM, Chiarion-Sileni V, Grob JJ, Dummer R, Wolchok JD, Schmidt H, Hamid O, Robert C, Ascierto PA, Richards JM, Lebbé C, Ferraresi V, Smylie M, Weber JS, Maio M, Konto C, Hoos A, de Pril V, Gurunath RK, de Schaetzen G, Suciu S, Testori A: Adjuvant ipilimumab versus placebo after complete resection of high-risk stage III melanoma (EORTC 18071): a randomized, double-blind, phase 3 trial. Lancet Oncol 2015;16:522-530. 